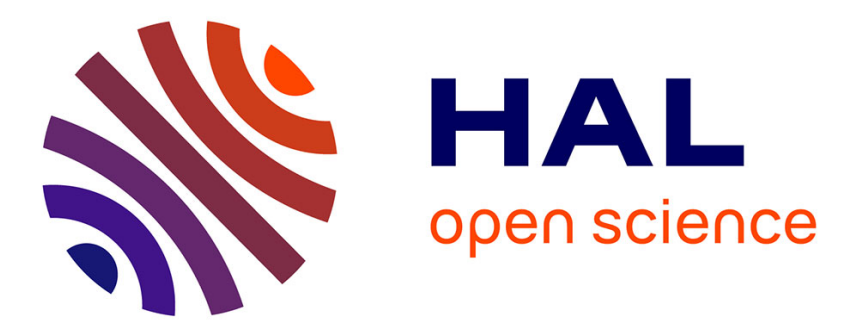

\title{
Lidar-relevant radiative properties of soot fractal aggregate ensembles
}

Lucas Paulien, Romain Ceolato, Laurent Soucasse, Franck Enguehard, Anouar Soufiani

\section{- To cite this version:}

Lucas Paulien, Romain Ceolato, Laurent Soucasse, Franck Enguehard, Anouar Soufiani. Lidarrelevant radiative properties of soot fractal aggregate ensembles. Journal of Quantitative Spectroscopy and Radiative Transfer, 2020, 241, pp.106706. 10.1016/j.jqsrt.2019.106706 . hal-02349170

\author{
HAL Id: hal-02349170 \\ https://hal.science/hal-02349170
}

Submitted on 14 Feb 2020

HAL is a multi-disciplinary open access archive for the deposit and dissemination of scientific research documents, whether they are published or not. The documents may come from teaching and research institutions in France or abroad, or from public or private research centers.
L'archive ouverte pluridisciplinaire HAL, est destinée au dépôt et à la diffusion de documents scientifiques de niveau recherche, publiés ou non, émanant des établissements d'enseignement et de recherche français ou étrangers, des laboratoires publics ou privés. 


\title{
Lidar-relevant radiative properties of soot fractal aggregate ensembles
}

\author{
Lucas Paulien $^{\mathrm{a}, \mathrm{b}}$, Romain Ceolato ${ }^{\mathrm{a}, *}$, Laurent Soucasse ${ }^{\mathrm{b}}$, Franck \\ Enguehard $^{\mathrm{c}}$, Anouar Soufiani ${ }^{\mathrm{b}}$ \\ ${ }^{a}$ ONERA, The French Aerospace Lab, Université de Toulouse, FR 31055, France \\ ${ }^{b}$ Laboratoire EM2C, CentraleSupélec, Université Paris-Saclay, Gif-sur-Yvette, 91192, \\ France \\ ${ }^{c}$ Institut PPrime, CNRS, Université de Poitiers, ISAE-ENSMA, 86962, Futuroscope, \\ Chasseneuil, France
}

\begin{abstract}
This study presents the averaged lidar-relevant radiative properties of numerically generated soot fractal aggregate ensembles. The radiative properties of these aggregates have been computed using the Superposition TMatrix Method, with emphasis put on those that are most relevant to lidar application; the backscattering and extinction cross-sections, the Lidar Ratio and the Linear Depolarization Ratio. These lidar-relevant radiative properties have been computed over a broad spectrum, going from the ultraviolet to the near infrared, in order to address lidar instrument need for a priori knowledge in signal inversion procedures and measurement analysis. By averaging the computed radiative properties according to each set of morphological parameters, we obtain statistically representative results and we study the impacts of morphological changes on these lidar-relevant radiative properties. Our results show a strong impact of the primary particle radius on all considered radiative properties, while the number of primary particles induce significant variations on the cross-sections only. The fractal dimension, although being an essential morphological parameter, has a weak influences on the lidar-relevant radiative properties.
\end{abstract}

Keywords: Soot Fractal Aggregates, lidar, Aerosol radiative properties, Modelling, T-Matrix

\footnotetext{
${ }^{*}$ Corresponding author : romain.ceolato@onera.fr
} 


\section{Introduction}

Soot particles are fine matter aerosols emitted from both natural and anthropogenic combustion processes, such as biomass burning or aeronautical engines. Freshly emitted soot particles are composed of clustered spherical primary particles, called monomers, which are formed during the incomplete combustion of organic materials. The structures resulting from the aggregation of primary particles are complex, and are often referred to as fractal-like shape $[1,2]$. The molecular composition as well as the sub-micronic size of these particles are problematic for human health, being cause of lung and artery diseases or cancers [3]. Moreover these particles can act as cloud condensation nuclei leading to the formation of condensation trails along plane tracks at high altitude [4]. Soot particles are also characterised by their high imaginary part of the optical index, therefore having a strong ability to absorb light in a wide spectrum.

These properties influence global climate by increasing the radiative forcing with both direct and indirect effects $[5,6]$. Both impacts on air quality and climate of soot particles are related to their morphology [3, 7]. Indeed, these Soot Fractal Aggregates (SFA) present various sizes and shapes according to the process of combustion that has led to their formation [8]. The characterisation of the morphological properties of emitted SFAs is therefore needed to evaluate and study the effect of these particles on climate and health. Several instrumental techniques are used to study these aggregates. Ex situ techniques such as electron microscopy can be used in order to precisely characterise the morphology of soot emitted from flames [9], but this method requires sampling procedures which can produce morphological changes and also requires extensive use in order to obtain statistically representative results. In situ techniques have the advantage of being nonintrusive, eliminating the possible bias induced by sampling. For example, X-ray Photoelectron Spectroscopy and Near Edge X-Ray Absorption Fine Structure have been used in order to study the composition and oxidation properties of airborne soot [10]. Another method is the Laser Induced Incandescence technique, which can be used to retrieve the soot volume fraction or the soot mass concentration profiles [11], or to determine the primary particle size [12]. Angular light scattering measurements are also able to measure the size distribution of SFAs [13].

To our knowledge, one limitation of these in situ instrumental techniques is their current inapplicability to study soot particles in atmospheric envi- 
ronment while keeping their non-intrusive aspect. To study soot particles in atmospheric conditions, remote sensing techniques are especially of interest. Light Detection And Ranging (lidar) instruments rely on the collection and detection of the light backscattered by the particles and molecules inside the volume of an emitted laser pulse in order to study this interacting medium. The detection of this backscattered light and the analysis of the associated signal can provide information on the particles, such as their concentration, size distribution or morphological properties $[14,15]$. These systems have already proven their ability to characterise and qualify different types of aerosols $[16,17]$ from remote area.

Because of the underdetermination of the problem associated with lidar measurements, the processing of the signals provided by these instruments relies on a priori knowledge of the radiative properties of the medium under study. This has led to the measurement and modelling of different types of airborne particle radiative properties with procedures that are in need of continuous improvement. The backscattering and extinction cross-sections, the Linear Depolarization Ratio (LDR) as well as the Lidar Ratio (LR) are especially useful to process and analyse lidar measurements. The backscattering and extinction cross-sections are directly involved in the inversion methods allowing to retrieve an aerosol concentration, while the LDR is often used as a discriminating parameter in order to characterise the airborne particle type. The Lidar Ratio is also a crucial parameter in the inversion algorithms of lidar signals [18].

The main objectives of this study are to quantify these radiative properties for statistically representative ensembles of SFAs and to study the effects induced by morphological changes. In this sense, we are interested in the light-matter interaction processes, in the framework of lidar application. After introducing this framework, we describe the models used to numerically generate SFAs and to calculate their radiative properties. Following the fractal model, SFAs are generated with a variety of morphological parameters. Statistically representative ensembles of SFAs are generated in order to evaluate the standard deviation of our results. Using the Multi-Sphere T-Matrix code [19], we chose to compute these lidar-relevant radiative properties from 300 to $1100 \mathrm{~nm}$, allowing to address a wide range of lidar instruments. After presenting our results, we discuss the impacts of the morphological parameters on these properties. 


\section{Lidar framework}

Polarization-resolved lidar are instruments able to measure simultaneously several states of polarization of the received backscattered light. The most common type of polarization-resolved lidar instruments can record the co-polarized $(\|)$ and cross-polarized $(\perp)$ components of the backscattered light relative to the emitted beam polarization plane. The elastic lidar equation under the single-scattering approximation provides the range-resolved backscattered power $P(r)$ at a given range $r$ from the receiver as:

$$
P_{\|, \perp}(r, \lambda)=K_{\|, \perp}(r, \lambda) O(r, \lambda) U_{\|, \perp}(r, \lambda)
$$

where the $\|, \perp$ subscripts refers to the state of polarization of the received light relative to the emitted pulse, $K(r, \lambda)$ is the lidar instrument function and $O(r, \lambda)$ is the range-dependent overlap function. $U(r, \lambda)$ is the attenuated backscattering function defined as :

$$
U_{\|, \perp}(r, \lambda)=\beta_{\|, \perp}(r, \lambda) \exp \left(-2 \int_{0}^{r} \alpha\left(r^{\prime}, \lambda\right) \mathrm{d} r^{\prime}\right)
$$

77

where $\beta(r, \lambda)$ and $\alpha(r, \lambda)$ are the backscattering and extinction coefficients, also referred to as lidar products, respectively defined as:

$$
\begin{aligned}
\beta_{\|, \perp}(r, \lambda) & =\sum_{i=m, p} \beta_{\|, \perp, i}(r, \lambda) \\
\beta_{\|, \perp, p}(r, \lambda) & =\int_{0}^{A_{\max }} n_{p}(a, r) C_{b a c k, \|, \perp}(a, \lambda) d a
\end{aligned}
$$

$$
\alpha_{p}(r, \lambda)=\int_{0}^{A_{\max }} n_{p}(a, r) C_{e x t}(a, \lambda) d a
$$

The subscripts $m$ and $p$ refer respectively to the molecular and particular contributions, $a$ is a characteristic length of the particles ranging from 0 to $A_{\max }$, and $n_{p}$ is the particle size distribution. The quantities $C_{b a c k}$ and $C_{\text {ext }}$ are the wavelength and size dependent backscattering and extinction cross-sections respectively, and are specific to the type, size, morphology and complex optical index of the particles. 
In the case of SFAs being present in the scattering volume, Equations (3) and (4) are hardly applicable. Indeed, SFAs are non-spherical and highly irregularly shaped particles, so that the definition of a characteristic length $a$ is a complex endeavour [20]. In the simplest case, if we consider a scattering volume containing exclusively a monodisperse distribution of SFAs, meaning that all particles have exactly the same morphology, the integral formulation of the particular backscattering and extinction coefficients in Equations (3) and (4) can be reformulated as :

$$
\beta_{\|, \perp, \text { mono }}(r, \lambda)=\frac{3 f_{v}(r)}{4 \pi N_{m} r_{m}^{3}} C_{\text {back }, \|, \perp}\left(r_{m}, N_{m}, \lambda\right)
$$

$$
\alpha_{\text {mono }}(r, \lambda)=\frac{3 f_{v}(r)}{4 \pi N_{m} r_{m}^{3}} C_{\text {ext }}\left(r_{m}, N_{m}, \lambda\right)
$$

where $f_{v}$ is the soot volume fraction and $r_{m}$ and $N_{m}$ are the monomer radius and number of monomers per aggregate respectively. The soot volume fraction $f_{v}$ is defined as :

$$
f_{v}=N \frac{4}{3} \pi N_{m} r_{m}^{3}
$$

where $N$ is the number density of aggregates.

The cross-sections in Equations (5) and (6) might nevertheless also depend on other parameters, such as the fractal dimension which will be defined in Section 3.1 or any other ad hoc parameter describing the morphology of SFAs. The dependence of the cross-sections on these parameters will be implecitely assumed in the rest of this section in order to lighten the notations

The ratio of the cross-polarized and co-polarized signals gives the volume depolarization ratio :

$$
\delta_{v}(r, \lambda)=\frac{K_{\perp}(r, \lambda)}{K_{\|}(r, \lambda)} \frac{\beta_{\perp}(r, \lambda)}{\beta_{\|}(r, \lambda)}
$$

Equations (3) to (6) link the retrievable lidar products to the radiative properties of individual particles. In order to process these lidar products, a priori knowledge on the particle radiative properties is necessary, as, in the general case, neither the size distribution (volume fraction in case of monodisperse SFAs) or morphology are known. 
Considering randomly oriented particles, the scattering properties in the backward direction of these particles are expressed using the random orientation scattering matrix $[21,22]$. In the backward direction, this matrix is diagonal :

$$
\boldsymbol{F}(\theta=\pi, \lambda)=\left(\begin{array}{cccc}
F_{11}(\pi, \lambda) & 0 & 0 & 0 \\
0 & F_{22}(\pi, \lambda) & 0 & 0 \\
0 & 0 & F_{33}(\pi, \lambda) & 0 \\
0 & 0 & 0 & F_{44}(\pi, \lambda)
\end{array}\right)
$$

This matrix provides the description of the backscattered light from the corresponding particles. Using this matrix, radiative properties convenient to lidar application are constructed, namely the unpolarized backscattering cross-section $C_{b a c k}$, the Lidar Ratio (LR) and the Linear Depolarization Ratio (LDR) $\delta$. Hereafter, we use the generic term lidar cross-sections to refer to the backscattering and extinction cross-sections, while the LR and LDR are named as lidar parameters, being ratios of cross-sections.

Considering a scattering medium with negligible molecular contribution $\left(\beta_{\|, \perp, m}<<\beta_{\|, \perp, p}\right.$ and $\left.\alpha_{m}<<\alpha_{p}\right)$, these lidar-relevant radiative properties are expressed as :

$$
C_{b a c k}(\lambda)=\frac{\lambda^{2}}{4 \pi^{2}} F_{11}(\pi, \lambda)=C_{b a c k, \|}(\lambda)+C_{b a c k, \perp}(\lambda)
$$

$$
L R(\lambda)=\frac{C_{\text {ext }}(\lambda)}{C_{\text {back }}(\lambda)}
$$

$$
\delta(\lambda)=\frac{C_{b a c k, \perp}(\lambda)}{C_{b a c k, \|}(\lambda)}=\frac{F_{11}(\pi, \lambda)-F_{22}(\pi, \lambda)}{F_{11}(\pi, \lambda)+F_{22}(\pi, \lambda)} \propto \delta_{v}
$$

The expressions of these lidar-relevant radiative properties in Equations (10), (11) and (12) are directly useful in the exploitation of the lidar products according to Equations (3) and (4).

The Lidar Ratio (LR) is a lidar parameter which quantifies the ratio of the extinguished light relatively to the backscattered light. It is a convenient parameter to express the potential backscattered signal obtained by a lidar instrument, and is often used in lidar signal inversion methods such as the Klett-Fernald's algorithm [18]. The Linear Depolarization Ratio (LDR) is often used in lidar applications as a discriminating parameter 
in order to classify the particles inside the measurement volume. This lidar parameter strongly depends on the morphology of the scatterer. Indeed, spherical particles scattering matrix elements are simplified by the relationship $F_{11}(\pi)=F_{22}(\pi)$. According to Equation (12), this relationship directly results in a null LDR for spherical particle. Hence, the LDR provides information on the non-sphericity of a particle. In lidar application, it can be compared to the volume $\operatorname{LDR}\left(\delta_{v}\right)$ to qualitatively specify which type of particles is present in the scattering volume.

\section{Methodology}

\subsection{Generation of soot fractal aggregates ensembles}

Soot aggregates are highly irregularly-shaped particles which are composed of clustered spherical primary particles, also called monomers. These particles are to be distinguished from black carbon particles, which refers to any particle with a high light absorption capacity and a high carbon content [23]. In this article, we focus on freshly emitted soot aggregates. These particles can be described using the fractal concept Forrest and Witten Jr [1] which provides a convenient formulation of their morphologies. In this model, the number of monomers is linked to the overall size of the aggregate with the power-law :

$$
N_{m}=k_{f}\left(\frac{R_{g}}{r_{m}}\right)^{D_{f}}
$$

where $N_{m}$ is the number of monomers, $r_{m}$ is the monomer radius, $R_{g}$ is the radius of gyration, $k_{f}$ is the fractal prefactor and $D_{f}$ is the fractal dimension. The radius of gyration $R_{g}$ is a quantity that represents the overall radius of an aggregate and is defined as :

$$
R_{g}=\sqrt{\frac{1}{N_{m}} \sum_{i=1}^{i=N_{m}} x_{i}^{2}}
$$

where $x_{i}$ is the distance from the $\mathrm{i}^{\text {th }}$ monomer center to the center of mass of the aggregate. The fractal prefactor $k_{f}$ is thought to be related to the local packing of the monomers $[24,25]$. The fractal dimension $D_{f}$ is a parameter the value of which lies between 1 and 3 and which provides information on the overall morphology of an aggregate. Indeed, as the fractal dimension decreases, the corresponding aggregate presents a more and more linear 
structure, while a compact aggregate is described by a fractal dimension approaching 3.

Various values of the monomer radius have been reported in the literature, ranging from $10-25 \mathrm{~nm}[26,27]$ up to $40-50 \mathrm{~nm}$ mean radius in large pool fires $[28,29]$. The reported values of the fractal dimension and fractal prefactor are also variable, the former being usually comprised between $D_{f}=$ 1.6 and $D_{f}=2.0$ [30], but they are predominantly found to be close to $D_{f} \approx 1.8$ with a fractal prefactor of $k_{f} \approx 1.3$ [31].

The fractal aggregates used in this study have been generated using the tunable Cluster-Cluster aggregation algorithm developed by Mackowski $[32,33]$. This algorithm allows the generation of the monomer positions for an aggregate while fixing the values of the fractal parameters $k_{f}$ and $D_{f}$, as well as the number of monomers $N_{m}$. Twenty-seven ensembles of one hundred SFAs have been generated using this algorithm, with the fractal prefactor always set to $k_{f}=1.3$ and the number of monomers to $N_{m}=45$, 125 or 450 . The fractal dimension has been set to either $D_{f}=1.6,1.8$ or 2.0 and the monomer radius to $r_{m}=10 \mathrm{~nm}, 20 \mathrm{~nm}$ or $40 \mathrm{~nm}$. Examples of generated aggregates are shown in Figure 1. Using Equation (14), we calculated the radius of gyration of each aggregate. When averaging these radii over ensembles of same morphological parameters, we found less than $0.3 \%$ deviation from the mean value of the radius of gyration. The average results are represented by the markers on Figure 1. 


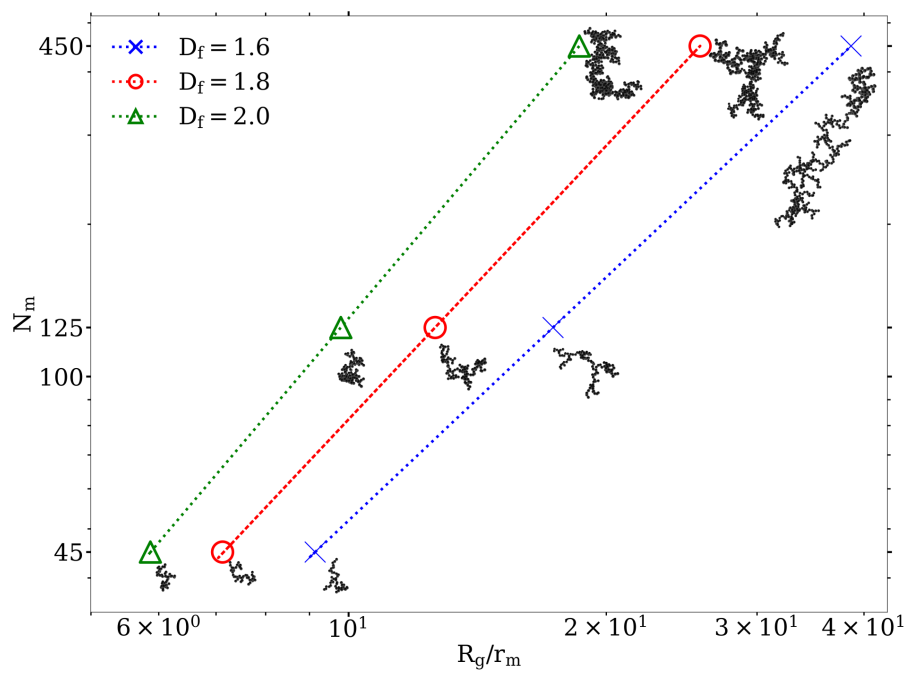

Figure 1: Number of monomers against the radius of gyration normalized by the monomer radius for ensembles of fractal dimension $D_{f}=1.6,1.8$, and 2.0. Each marker represents one of the generated aggregate ensembles and the dashed lines are the fitted curves using equation 13. The fitting parameters are $D_{f}=1.5975 \pm 0.0002$ and $k_{f}=1.3127 \pm 0.0009$ (blue curve, cross), $D_{f}=1.7968 \pm 0.0002$ and $k_{f}=1.3141 \pm 0.0010$ (red curve, circles), $D_{f}=1.9965 \pm 0.0002$ and $k_{f}=1.3142 \pm 0.0008$ (green curve, triangles). Examples of the aggregate morphologies are represented nearby the corresponding markers. In these representations, the monomer radius is set to the same arbitrary value, and the aggregates have been orientated so that the longest vector linking any couple of monomer centers is placed in the plane of the figure.

The fractal parameters $D_{f}$ and $k_{f}$ of each ensemble have been retrieved by fitting the number of monomers against the radius of gyration normalized by the monomer radius using Equation (13). The relative discrepancy between initial and retrieved values of the fractal parameters are less than $1.5 \%$, hence being in very good agreement as shown in Figure 1. Hereafter, the initial values are used in order to lighten the notations.

Although the aggregates of each ensemble can be characterised by the same morphological parameters, each aggregate still presents its own disposition of the monomers in space. These different morphologies within the same ensemble can result in variations of the radiative properties. Indeed, previous studies have highlighted the importance of using ensemble averaging as well as orientation averaging [34]. In our study we compute random-orientation radiative properties, and then we average these properties over the one hundred aggregates of each ensemble. Results (not presented in this paper) 
show that as we increase the number of aggregates in the ensembles from one to around fifty, there can be strong variations of the standard deviation at certain wavelengths. By adding more aggregates into the ensembles, these variations attenuate. We chose to use ensembles of one hundred aggregates as a compromise between representativity of the aggregate morphologies and their associated radiative property standard deviation, and the computation time and resources.

\subsection{Refractive index of soot particles}

The computation of the radiative properties also requires the material complex optical index. Variable values of the real and imaginary parts of the refractive index of soot particles have been reported in the literature, under different conditions of emission, soot temperature, fuel, wavelength and determination method $[35,36,37]$. As our intent is to provide representative values of the lidar-relevant radiative properties on a large spectrum $(300 \mathrm{~nm} \leq$ $\lambda \leq 1100 \mathrm{~nm}$, with a wavelength step $\Delta \lambda=20 \mathrm{~nm}$ ), we used the wavelengthdependent dispersion law from Chang and Charalampopoulos [38], as shown in Figure 2.

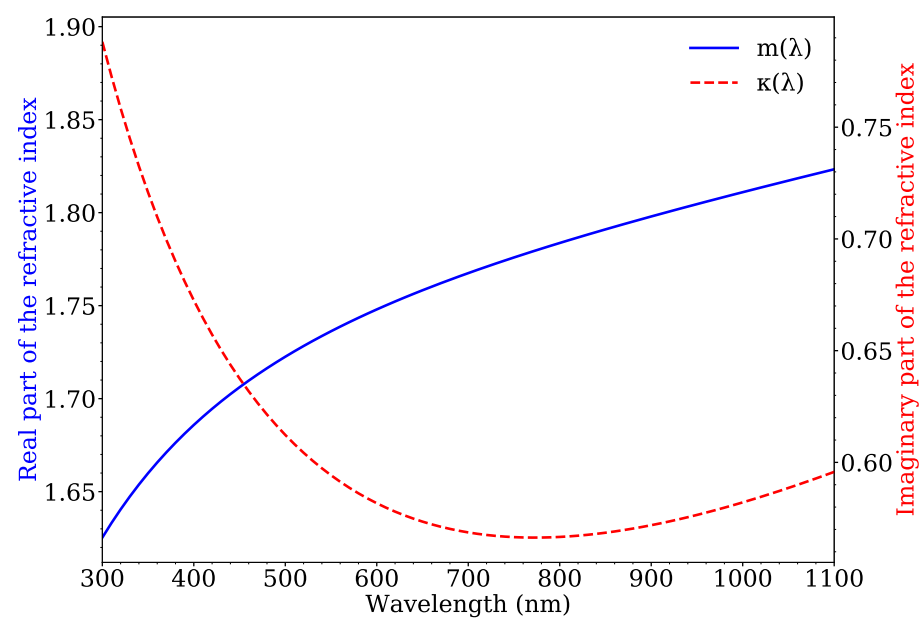

Figure 2: Refractive index $m(\lambda)=n(\lambda)+i \kappa(\lambda)$ used in this study [38].

\subsection{Modelling of the lidar-relevant radiative properties}

Different methods can be used to model the radiative properties of SFAs. The well known Lorenz-Mie theory can be used to calculate the radiative 
properties of spheres [39], but this method has shown poor accuracy when applied to SFAs because of the use of non-representative equivalent spheres $[20,40]$. The Rayleigh-Debye-Gans for Fractal Aggregates (RDG-FA) theory is another method which is specifically designed to compute the radiative properties of fractal aggregates [41]. This approximation provides good angle integrated results, e.g. the extinction cross-sections. However, the angle dependent results obtained using RDG-FA such as the scattering matrix elements can be inaccurate, especially for high primary particle size parameter [42]. The Discrete Dipole Approximation method has also been used on realistic aggregate morphologies $[43,44,45]$. This method provides quantification of the scattered field from a particle of any shape illuminated by any incoming field. The limitation of this method comes from its computationally intensive character, especially if orientation averaged and ensemble averaged results are sought. The Superposition T-Matrix (STM) method is commonly used for the computation of SFA radiative properties [46, 47]. This method also shows limitations, as the scatterer must be composed of strictly non-overlapping spheres, preventing the computation of complex morphological properties such as sphere overlapping, necking or complex coating phenomenon. This applicability criterion is met by the fractal aggregate model described in Section 3.1.

The STM method consists in expressing the scattered field by the overall aggregate as the superposition of the partial fields contributed by each individual sphere, taking into account the internal multiple scattering between spheres. As a detailed description of this method and of the mathematics involved is available in the papers from Mackowski and Mishchenko [48], we only provide a short description of the principles of this method hereafter. The external incoming field and outcoming field of each sphere are expanded into Vector Spherical Wave Functions centered about the sphere origins, and the associated expansion coefficients are calculated. The transition matrix (T-Matrix) transforms the outcoming field centered about one sphere origin into the incoming field of another sphere. This allows the computation of the internal multiple scattering among monomers, i.e. the way the scattered field from one sphere impacts the others. The sets of equations involved are iteratively solved until a specified convergence criterion is met regarding the radiative properties of each sphere, and the sphere-centered T-matrices are then transformed into a single cluster-centered T-matrix. This T-matrix allows the computation of the coefficients of the far-field scattered transverse spherical wave, which in turn leads to the computation of the amplitude 
scattering matrix. By applying the linearized form of the two sets of rotation functions to (i) first the T-Matrix to compute the scattering coefficients; (ii) second the amplitude scattering matrix to compute the orientation averaged amplitude scattering matrix, simplification of the random orientation scattering matrix calculation is achieved. The random orientation extinction cross-section is also calculated from the cluster-centered T-Matrix. In our study, we used the Multi-Sphere T-Matrix (MSTM) code [19].

\section{Numerical results}

Using the MSTM code, each individual SFA's lidar-relevant radiative properties have been calculated. Afterwards, the SFA's radiative properties have been averaged over their corresponding morphological ensemble with the same number of monomers $N_{m}$, monomer radius $r_{m}$ and fractal dimension $D_{f}$. 


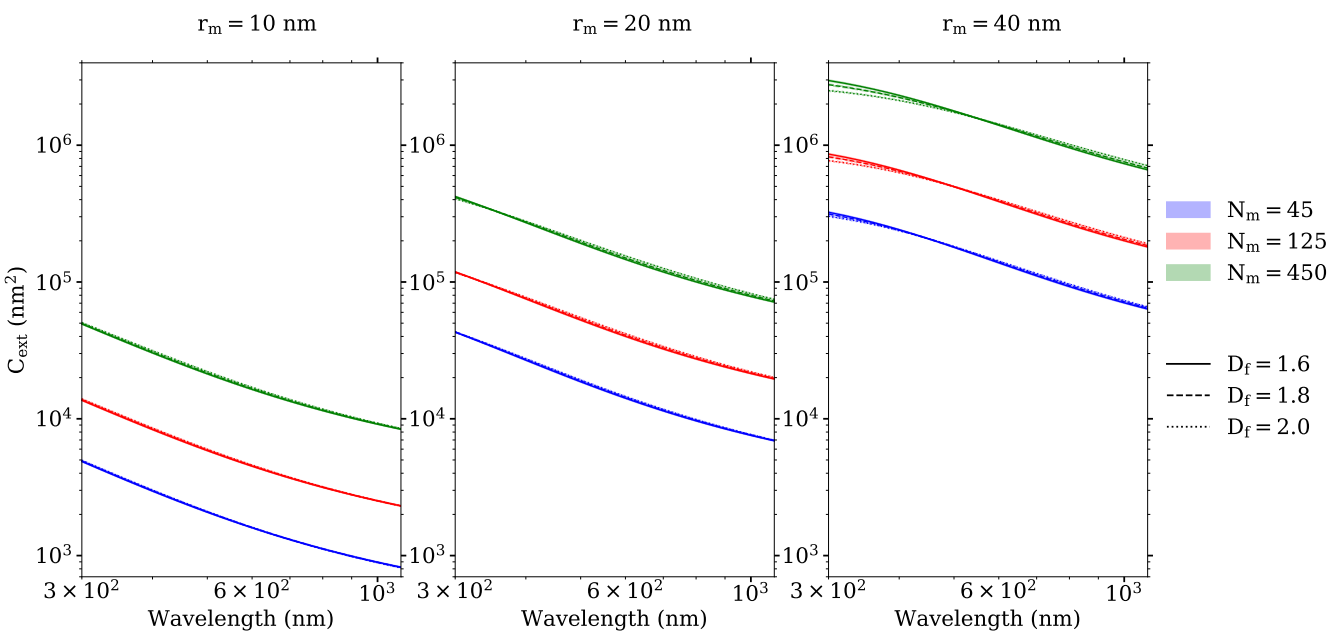

(a) Extinction cross-sections.

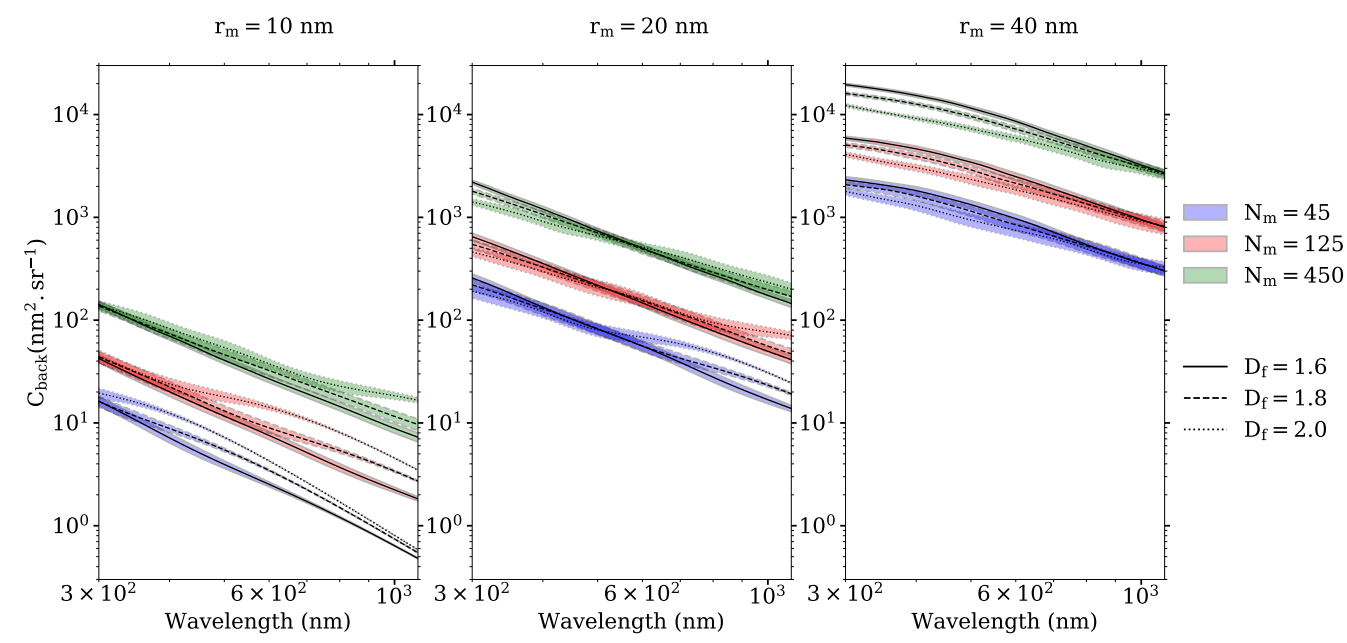

(b) Backscattering cross-sections.

Figure 3: Extinction (a) and backscattering (b) cross-sections of SFA ensembles. The three panels are ordered according to the SFA ensemble monomer radius, i.e. $10 \mathrm{~nm}$, $20 \mathrm{~nm}$, and $40 \mathrm{~nm}$ monomer radius from left to right. The blue, red and green curves are associated with aggregates composed of 45,125 , and 450 monomers respectively. The coloured stripes represent the standard deviations to the mean values of the cross-sections. The solid, dashed, and dotted lines represent different values of the fractal dimension, i.e. $D_{f}=1.6, D_{f}=1.8$, and $D_{f}=2.0$ respectively. The fractal prefactor of all aggregates is set to $k_{f}=1.3$. 
According to Figure 3a, the extinction cross-sections present a consistent spectral and morphological dependence across all ensembles. The extinction cross-sections are decreasing with wavelength, and increasing with monomer radius. Higher number of monomers also results in higher cross-sections. Fractal dimension variations produce a negligible effect on the extinction cross-section, with the exception of the largest aggregate ensembles (i.e. $\left.r_{m}=40 \mathrm{~nm}, N_{m}=450\right)$. In these cases, higher fractal dimensions result in lower extinction cross-sections in the UV part of the spectrum. The standard deviations of the extinction cross-sections across all wavelength and ensembles are below $0.1 \%$.

As shown in Figure 3b, the backscattering cross-sections are also decreasing with wavelength, with a standard deviation of about $10 \%$ over the whole spectrum for each ensemble of aggregates. A difference up to more than one order of magnitude between the backscattering cross-sections in the UV and in the NIR can be observed. This decrease with wavelength is more important for smaller aggregates, i.e. as the monomers are few and small. Comparing the Figures 3a and 3b, it is apparent that the backscattering cross-sections are decreasing more rapidly with wavelength than the extinction cross-sections.

For the same other morphological parameters, doubling the monomer radius leads to a backscattering cross-section increase by a factor ranging from 10 in the UV part of the spectrum up to 50 in the NIR part. The backscattering cross-section decrease with wavelength is greater for smaller monomer radius. Hence, the monomer radius of the aggregates also influences the wavelength dependence of the radiative properties.

Increasing tenfold the number of monomers induces a 6 to 12 times increase of the backscattering cross-section when the monomer radius is equal to either $r_{m}=20 \mathrm{~nm}$ or $r_{m}=40 \mathrm{~nm}$. For monomer radius $r_{m}=10 \mathrm{~nm}$, this factor ranges from 7 to 29 . This difference for smaller monomers is most certainly due to the different spectral dependence of the backscattering cross-sections. Indeed, the wavelength dependence of the backscattering cross-sections is also influenced by the number of monomers, with lower number of monomers inducing a steeper decrease. The $10 \mathrm{~nm}$ radius ensembles in particular present the strongest variation.

The impact of the fractal dimension on the backscattering cross-section is interdependent on the monomer radius and number. Indeed, for small monomer radius as represented on the left panel of Figure 3b, the compact aggregates with higher fractal dimension present higher backscattering cross- 
sections. In this panel, SFAs with few monomers, i.e. $N_{m}=45$, present a noticeable separation of the curves for different fractal dimensions (blue curves) from the UV part to the NIR, while curves associated with higher numbers of monomers are separated at higher wavelengths. For $r_{m}=20 \mathrm{~nm}$, linear SFAs present higher backscattering cross-sections in the UV part of the spectrum, and conversely in the NIR part. SFA ensembles of $r_{m}=40 \mathrm{~nm}$ monomer radius present increasingly ordered backscattering cross-sections with decreasing fractal dimension.

Some features of the different curves seem to be shifted at larger wavelengths when increasing the overall size of the aggregates. For example, on the left panel of Figure 3b, the separation of the curves of different fractal dimensions occurs at larger wavelengths with higher monomer radius and/or number. The considered complex optical index varying only slightly in this spectrum, this indicates that a parameter similar to the usual size parameter $x=2 \pi a / \lambda$ could be driving the wavelength dependence of these radiative properties. However, the length scale involved still needs to be defined, and this study is out of the scope of this article.

\subsection{Lidar Ratio}

As the LR is the ratio between the extinction cross-section and the backscattering cross-section, both morphological and spectral dependence of the LR are closely related to those of the lidar cross-sections. Indeed, Figure 4 shows an increasing LR with larger wavelengths, which is consistent with the wavelength dependence of the lidar cross-sections. 


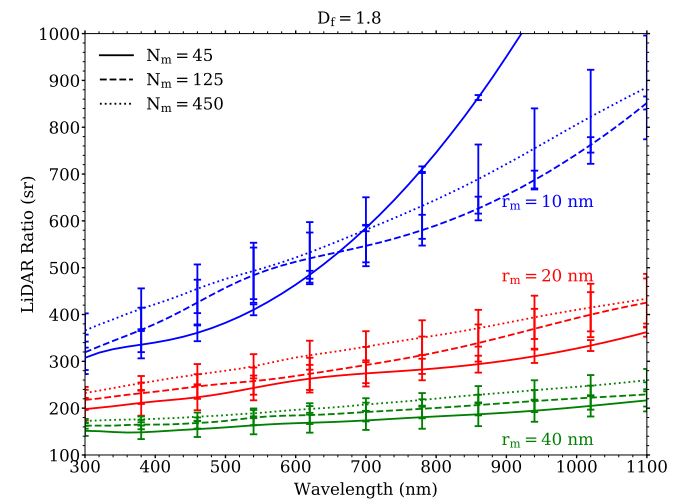

(a) LR variation with monomer radius and number

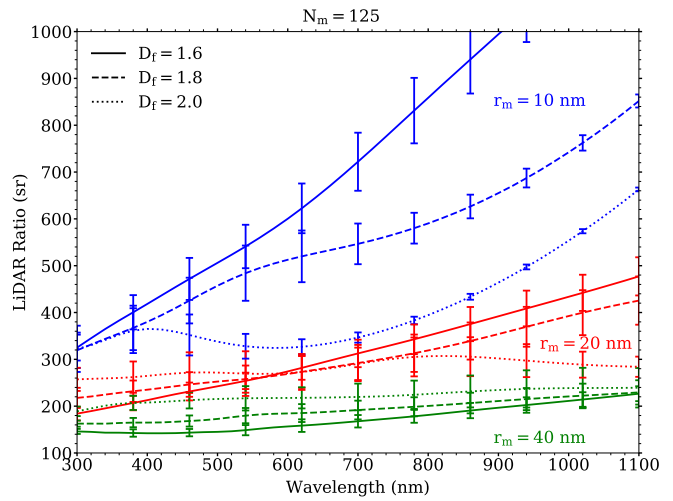

(b) LR variation with monomer radius and fractal dimension

Figure 4: LR of SFA ensembles. The three sets of coloured curves are ordered according to the SFA ensemble monomer radius, i.e. $10 \mathrm{~nm}, 20 \mathrm{~nm}$, and $40 \mathrm{~nm}$ monomer radius for the blue, red and green curves respectively. The full, dashed and dotted lines are associated with aggregates composed of 45, 125, and 450 monomers respectively on Figure (a), and with $D_{f}=1.6,1.8$, and 2.0 on Figure (b). The error bars are plotted every four points and represent the standard deviations to the mean values of LRs. On Figure (a), the fractal dimension in set to $D_{f}=1.8$, and on Figure (b) the number of monomers is set to $N_{m}=125$. The fractal prefactor of all aggregates is set to $k_{f}=1.3$.

Similarly, larger monomer radius reduces the LR, which is consistent with the increasing backscattering cross-section presented in Figure 3b. LR values with different monomer radius show close values in the UV part of the spectrum, and diverge in the near infrared. Aggregates with smaller monomer radius present a more important increase of the LR. This relationship is also in accordance with the spectral dependence of the backscattering cross-section in Figure 3b.

Higher number of monomers also induces higher LR, with the noticeable exception of the ensemble of smallest aggregates (i.e. $r_{m}=10 \mathrm{~nm}, N_{m}=$ 45). This ensemble wavelength dependence is most probably due to the steeper decrease of the backscattering cross-section of this ensemble as shown in Figure 3b (left panel; blue dashed line). The variation of the LR with SFA's number of monomers is still small, the associated standard deviations overlapping on many if not all parts of the spectrum.

Impacts of the fractal dimension are harder to evaluate, as several distinctive trends occur as a function of both wavelength and monomer radius. Indeed, Figure $4 \mathrm{~b}$ shows that for small aggregates (blue curves), a more 


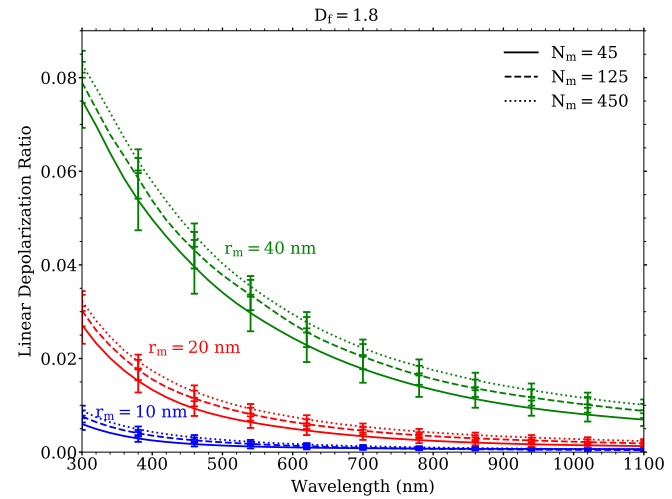

(a) LDR variation with monomer radius and number

\subsection{Linear Depolarization Ratio}

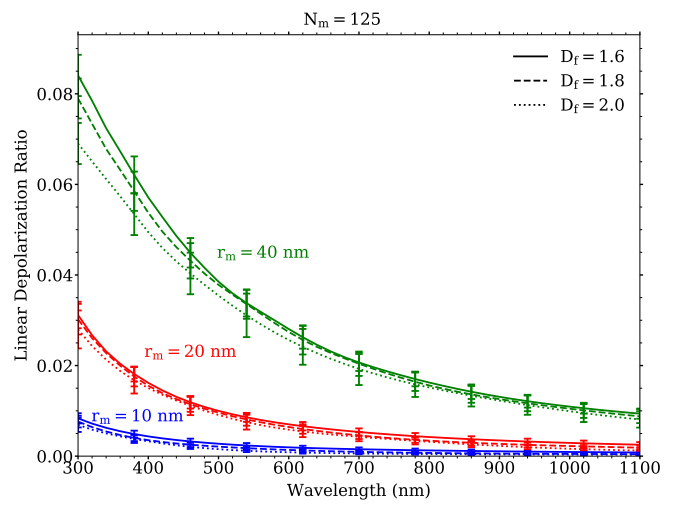

(b) LDR variation with monomer radius and fractal dimension

Figure 5: LDRs of SFA ensembles. The blue, red and green colours are used to differentiate SFA ensembles of $10 \mathrm{~nm}, 20 \mathrm{~nm}$, and $40 \mathrm{~nm}$ monomer radius respectively. The solid, dashed and dotted lines represent SFA ensembles of varying number of monomers and fractal dimension, i.e. $N_{m}=45, N_{m}=125$ and $N_{m}=450$, respectively for Figure (a) in which case the fractal dimension is set to $D_{f}=1.8$, and $D_{f}=1.6, D_{f}=1.8$, and $D_{f}=2.0$ respectively for Figure (b) with the number of monomers set to $N_{m}=125$. The fractal prefactor of all aggregates is set to $k_{f}=1.3$.

compact aggregate induces a lower LR, while large aggregates (green curves) present the inverse feature. Intermediate size (red curves) presents both trends, the former in the NIR part and the latter in the lower part of the spectrum. Similarly, the standard deviations of the LRs are predominantly affected by the backscattering cross-section standard deviations. 
LDR also increases with increasing number of monomers, although with a far less noticeable effect than monomer radius as shown in Figure 5a. A modification of the fractal dimension also induces small variations of the LDR, as presented in Figure 5b, lower fractal dimension inducing higher LDR values. This fractal dimension impact is amplified at larger monomer radius and smaller wavelength. At constant monomer radius, the LDR standard deviations overlap over the whole spectrum, for either different numbers of monomers or fractal dimensions.

\section{Discussion}

The monomer radius $r_{m}$ appears to be the morphological parameter that influences the most the considered lidar-relevant radiative properties, as they all show large variations according to $r_{m}$. The monomer number has a strong impact on the lidar cross-sections $\left(C_{\text {back }}\right.$ and $\left.C_{e x t}\right)$, and a weak impact on lidar parameters (LR and LDR). The fractal dimension, although being an essential parameter of the fractal model, only induces low variations on the lidar-relevant radiative properties. In specific cases, as for the LR of small monomer radius aggregates presented on the blue curves of Figure $4 \mathrm{~b}$, it can present a significant impact. In order to stay faithful to realistic SFA morphologies, the variation range of the fractal dimension has been kept low. Hence, our results don't exclude the possibility that very high or very low fractal dimensions, i.e. $D_{f} \approx 3$ or $D_{f} \approx 1$, might have larger impacts on these radiative properties.

The relatively strong dependence of the lidar cross-sections on the monomer radius and number seems to be related to the overall size of the aggregate, with larger geometrical cross-sections and volume of matter resulting in higher radiative cross-sections. In order to study this effect, we normalize the lidar cross-sections by $(4 / 3) \pi N_{m} r_{m}^{3}$, the volume occupied by a single aggregate, as in Equations (5) and (6). This effectively transforms these normalized cross-sections into the backscattering and extinction coefficients per soot volume fraction as shown in Equations (5) and (6). Results are presented in Figure 6. 


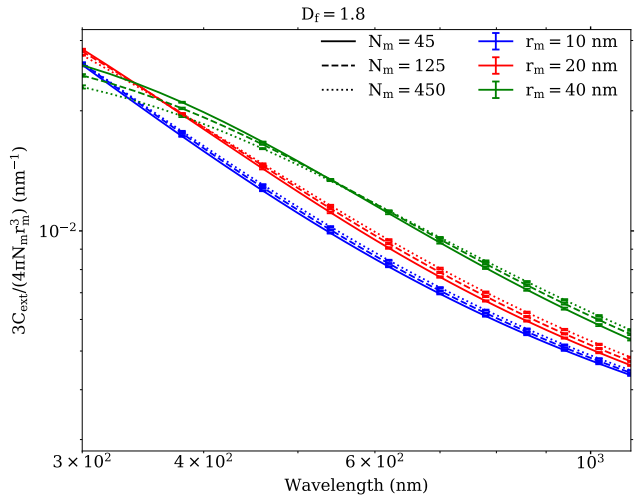

(a) Volume normalized extinction crosssection variation with monomer radius and number

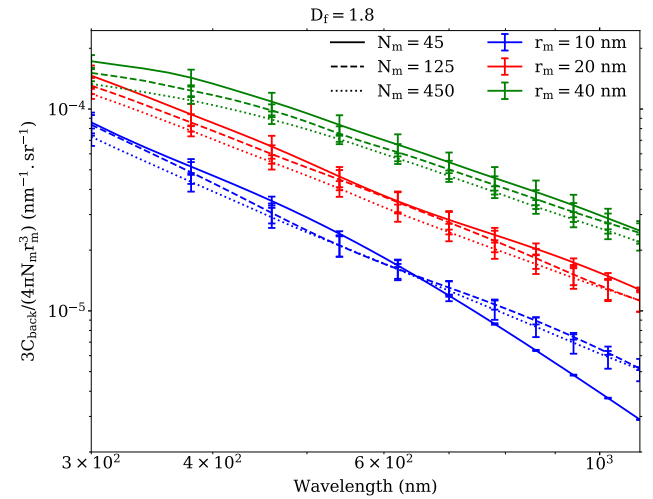

(b) Volume normalized backscattering cross-section variation with monomer radius and number

Figure 6: Volume normalized cross-sections of SFA ensembles. The three sets of coloured curves are ordered according to the SFA ensemble monomer radius, i.e. $10 \mathrm{~nm}, 20 \mathrm{~nm}$, and $40 \mathrm{~nm}$ monomer radius for the blue, red and green curves respectively. The full, dashed and dotted lines are associated with aggregates composed of 45, 125, and 450 monomers respectively. The error bars are plotted every four points and represent the standard deviations to the mean values of the volume normalized cross-sections. The fractal dimension is set to $D_{f}=1.8$ and the fractal prefactor of all aggregates is set to $k_{f}=1.3$.

According to Figure 6, the monomer number dependence of both lidar cross-sections is reduced by the normalization, but the monomer radius influence is still observable, especially in the backscattering cross-sections. Indeed, in the VIS to NIR part of the spectrum, increasing twofold the monomer radius produces a slight increase of the volume normalized extinction crosssection by 5 to $20 \%$, while the volume normalized backscattering crosssection is multiplied by a factor going from 1.5 up to 6 . This indicates that the link between backscattering and morphology in this spectrum can not be fully described by the volume of matter of an aggregate. On the other hand, the volume normalized extinction cross-section curves are much closer together, indicating that extinction is more of a volume driven process. This is also in agreement with the close extinction cross-sections values obtained using volume equivalent spheres [20]. Taking Equations (5) and (6), this implies that an increase of the soot volume fraction due to aggregates with larger monomers would not produce the same effect on the extinction and backscattering coefficients as an increase due to higher number of monomers. 
The spectral dependence of the LDR is consistent with the fact that as the wavelength increases, the illuminating wave is less sensitive to the nonsphericity of the SFA. Indeed, as previously mentioned, spherical particles do not depolarize the scattered light in the backward direction. As the wavelength increases, the relative size of the aggregates compared to wavelength is smaller, and light is less sensitive to the shape irregularities. This is further supported by the low LDR of the $r_{m}=10 \mathrm{~nm}$ SFA ensembles. The increasing LDR for lower fractal dimension, i.e. more linear SFAs, is consistent with the non-sphericity of an aggregate inducing more depolarization in the backward direction. The depolarization of soot fractal aggregate is a byproduct of the multiple scattering among primary particles [49], and is sensitive to the internal fine structure of the aggregates. By increasing the monomer radius, or equivalently reducing the wavelength, the LDR is increasingly sensitive to the fractal dimension. Still, these effects do not seems significant enough to estimate the fractal dimension using LDR measurements.

Even averaged over one hundred aggregates, the backscattering crosssection, LR and LDR standard deviations of ensembles of same morphological parameters are important. The LDR and the LR being dependent on the backscattering cross section, these statistical deviations indicate that the backscattering cross section is sensitive to the fine structure of the aggregates. As the fractal parameters are nearly constant in each ensemble, it can be assumed that this morphological description is not sufficient in order to precisely describe an aggregate morphology and the resulting lidarrelevant radiative properties. Moreover, SFAs emitted from real combustion processes have been found to present observable differences from the fractal model used in this study. Indeed, the monomers can present overlapping, necking effect, or lognormal size distribution [50]. During their ageing, these particles can also be subject to morphology and composition changes, such as those induced by necking or coating phenomena [24, 51]. While these morphological properties can influence the radiative properties of soot aggregates $[46,52,53]$, their impacts have not been investigated in this study, and SFAs are considered as composed of monodisperse spheres in point contact. The retrieval of soot morphological parameters through lidar measurements is a complex process, as the number of unknowns is potentially greater than the number of measurable quantities. Prior sensitivity studies would be required in order to describe in which magnitude a retrieval of soot morphological properties is possible, taking into account instrumental noise, polydispersion of any of the morphological parameters inside the measurement volume, and 
range dependent quantities. Still, our results show promising outlooks for lidar microphysical inversion, specifically for the retrieval of monomer radius. Knowledge of this morphological parameter could provide a first assessment of the LR values, likely allowing the use of an inverse algorithm in order to retrieve both backscattering and extinction coefficient profiles.

While the values of the computed lidar cross-sections and LDR are in good agreement with other modelled values found in the literature [46, 54], the LR values presented here are much higher than those usually used in lidar inversion methods [55]. Several factors can explain this discrepancy. As the interacting particles are part of a volume formed by the laser pulse divergence and length, soot can be mixed with other aerosols during lidar measurements, as in smoke. A second influencing factor could be the ageing status of the soot particles, as the atmospheric processing of these aerosols change their radiative properties. Considering the fractal model and the complex optical index dispersion law used in this study, our results are more relevant to freshly emitted SFAs, i.e. uncoated soot aerosols not yet influenced by ageing processes. Lidar measurements of freshly emitted soot particles would be of particular interest in order to compare our modelling results to experimental data, as, to our knowledge, there exist no such measurements in the literature.

\section{Conclusion}

This study aims at quantifying the radiative properties of Soot Fractal Aggregates that are most useful to process lidar measurements. Statistically representative ensembles of SFAs have been generated and results have been averaged over samples of one hundred aggregates of identical morphological parameters. In each ensemble, the morphology of any individual aggregate is still unique. This results in important standard deviations of the lidarrelevant radiative properties and supports the need for statistically representative ensembles. It also suggests that the fractal model is not self-sufficient in order to describe the morphology of fractal aggregates, and that other morphological parameters might be needed. Our objectives was also to quantify realistic wavelength dependence of the aggregate radiative properties. This was achieved by computing them over a wide spectrum and by considering a realistic wavelength dependent refractive index, although other values exist in the literature.

The main conclusions of this study are : 
- The monomer radius $r_{m}$ strongly influences all of the studied lidarrelevant radiative properties.

- The number of monomers per aggregate $N_{m}$ has also a strong effect on the lidar cross-sections, but has a limited impact on the lidar parameters such as the Lidar Ratio and the linear depolarization ratio.

- The aggregate fractal dimension $D_{f}$ has a weak impact on all lidarrelevant radiative properties.

These results are quite significant for lidar application, as the processing of the elastic lidar products (i.e. backscattering coefficients $\beta$, extinction coefficients $\alpha$ and volume depolarization ratio $\delta_{v}$ ) might provide means to evaluate the monomer radius.

\section{Acknowledgements}

This work has been partially funded by the doctoral school Sciences mécaniques et énergétiques, matériaux et géosciences (SMEMAG) and the ONERA, and was conducted in the framework of the PROMETE project. We would like to acknowledge Daniel W. Mackowski for making publicly available the MSTM code, as well as Matthew J. Berg and Christopher M. Sorensen.

\section{References}

[1] S. Forrest, T. Witten Jr, Long-range correlations in smoke-particle aggregates, Journal of Physics A: Mathematical and General 12 (1979) L109.

[2] Ü. Ö. Köylü, G. Faeth, T. L. Farias, M. d. G. Carvalho, Fractal and projected structure properties of soot aggregates, Combustion and Flame 100 (1995) 621-633.

[3] E. J. Highwood, R. P. Kinnersley, When smoke gets in our eyes: The multiple impacts of atmospheric black carbon on climate, air quality and health, Environment International 32 (2006) 560-566.

[4] B. Kärcher, Formation and radiative forcing of contrail cirrus, Nature communications 9 (2018) 1824. 
[5] O. Boucher, D. Randall, P. Artaxo, C. Bretherton, G. Feingold, P. Forster, V.-M. Kerminen, Y. Kondo, H. Liao, U. Lohmann, P. Rasch, S. Satheesh, S. Sherwood, B. Stevens, X. Zhang, Clouds and Aerosols, Cambridge University Press, Cambridge, United Kingdom and New York, NY, USA, p. 571-658.

[6] G. Myhre, D. Shindell, F.-M. Bréon, W. Collins, J. Fuglestvedt, J. Huang, D. Koch, J.-F. Lamarque, D. Lee, B. Mendoza, T. Nakajima, A. Robock, G. Stephens, T. Takemura, H. Zhang, Anthropogenic and Natural Radiative Forcing, Cambridge University Press, Cambridge, United Kingdom and New York, NY, USA, p. 659-740.

[7] R. K. Chakrabarty, N. D. Beres, H. Moosmüller, S. China, C. Mazzoleni, M. K. Dubey, L. Liu, M. I. Mishchenko, Soot superaggregates from flaming wildfires and their direct radiative forcing, Scientific reports 4 (2014) 5508.

[8] L. Tumolva, J.-Y. Park, J.-s. Kim, A. L. Miller, J. C. Chow, J. G. Watson, K. Park, Morphological and elemental classification of freshly emitted soot particles and atmospheric ultrafine particles using the tem/eds, Aerosol Science and Technology 44 (2010) 202-215.

[9] G. Okyay, E. Héripré, T. Reiss, P. Haghi-Ashtiani, T. Auger, F. Enguehard, Soot aggregate complex morphology: 3d geometry reconstruction by sem tomography applied on soot issued from propane combustion, Journal of Aerosol Science 93 (2016) 63-79.

[10] F.-X. Ouf, P. Parent, C. Laffon, I. Marhaba, D. Ferry, B. Marcillaud, E. Antonsson, S. Benkoula, X.-J. Liu, C. Nicolas, et al., First in-flight synchrotron x-ray absorption and photoemission study of carbon soot nanoparticles, Scientific reports 6 (2016) 36495.

[11] J. D. Black, M. P. Johnson, In-situ laser-induced incandescence of soot in an aero-engine exhaust: Comparison with certification style measurements, Aerospace Science and Technology 14 (2010) 329-337.

[12] H. Michelsen, C. Schulz, G. Smallwood, S. Will, Laser-induced incandescence: Particulate diagnostics for combustion, atmospheric, and industrial applications, Progress in Energy and Combustion Science 51 (2015) 2-48. 
[13] M. Bouvier, J. Yon, G. Lefevre, F. Grisch, A novel approach for in-situ soot size distribution measurement based on spectrally resolved light scattering, Journal of Quantitative Spectroscopy and Radiative Transfer 225 (2019) 58-68.

[14] D. Müller, F. Wagner, U. Wandinger, A. Ansmann, M. Wendisch, D. Althausen, W. von Hoyningen-Huene, Microphysical particle parameters from extinction and backscatter lidar data by inversion with regularization: experiment, Applied Optics 39 (2000) 1879-1892.

[15] A. Miffre, G. David, B. Thomas, P. Rairoux, Atmospheric non-spherical particles optical properties from uv-polarization lidar and scattering matrix, Geophysical Research Letters 38 (2011).

[16] A. H. Omar, D. M. Winker, M. A. Vaughan, Y. Hu, C. R. Trepte, R. A. Ferrare, K.-P. Lee, C. A. Hostetler, C. Kittaka, R. R. Rogers, et al., The calipso automated aerosol classification and lidar ratio selection algorithm, Journal of Atmospheric and Oceanic Technology 26 (2009) 1994-2014.

[17] S. Burton, R. Ferrare, C. Hostetler, J. Hair, R. Rogers, M. Obland, C. Butler, A. Cook, D. Harper, K. Froyd, Aerosol classification using airborne high spectral resolution lidar measurements-methodology and examples, Atmospheric Measurement Techniques 5 (2012) 73-98.

[18] J. D. Klett, Lidar inversion with variable backscatter/extinction ratios, Applied optics 24 (1985) 1638-1643.

[19] D. Mackowski, M. Mishchenko, A multiple sphere t-matrix fortran code for use on parallel computer clusters, Journal of Quantitative Spectroscopy and Radiative Transfer 112 (2011) 2182-2192.

[20] R. Ceolato, F. Gaudfrin, O. Pujol, N. Riviere, M. J. Berg, C. M. Sorensen, Lidar cross-sections of soot fractal aggregates: Assessment of equivalent-sphere models, Journal of Quantitative Spectroscopy and Radiative Transfer 212 (2018) 39-44.

[21] M. Mishchenko, J. Hovenier, Depolarization of light backscattered by randomly oriented nonspherical particles., Optics Letters 20 (1995) $1356-1358$. 
[22] M. I. Mishchenko, Electromagnetic scattering by particles and particle groups: an introduction, Cambridge University Press, 2014.

[23] P. Buseck, K. Adachi, A. Gelencsér, É. Tompa, M. Pósfai, Are black carbon and soot the same?, Atmospheric Chemistry and Physics Discussions 12 (2012) 24821-24846.

[24] W. R. Heinson, P. Liu, R. K. Chakrabarty, Fractal scaling of coated soot aggregates, Aerosol Science and Technology 51 (2017) 12-19.

[25] C. M. Sorensen, G. C. Roberts, The prefactor of fractal aggregates, Journal of colloid and interface science 186 (1997) 447-452.

[26] Ü. Ö. Köylü, G. M. Faeth, Structure of overfire soot in buoyant turbulent diffusion flames at long residence times, Combustion and Flame 89 (1992) 140-156.

[27] T. C. Bond, R. W. Bergstrom, Light absorption by carbonaceous particles: An investigative review, Aerosol science and technology 40 (2006) $27-67$.

[28] G. Mulholland, W. Liggett, H. Koseki, The effect of pool diameter on the properties of smoke produced by crude oil fires, in: Symposium (International) on Combustion, volume 26, Elsevier, pp. 1445-1452.

[29] K. A. Jensen, J. M. Suo-Anttila, L. G. Blevins, Measurement of soot morphology, chemistry, and optical properties in the visible and nearinfrared spectrum in the flame zone and overfire region of large jp- 8 pool fires, Combustion Science and Technology 179 (2007) 2453-2487.

[30] M. Lapuerta, J. Barba, A. D. Sediako, M. R. Kholghy, M. J. Thomson, Morphological analysis of soot agglomerates from biodiesel surrogates in a coflow burner, Journal of Aerosol Science 111 (2017) 65-74.

[31] C. Sorensen, The mobility of fractal aggregates: a review, Aerosol Science and Technology 45 (2011) 765-779.

[32] D. W. Mackowski, Electrostatics analysis of radiative absorption by sphere clusters in the rayleigh limit: application to soot particles, Applied optics 34 (1995) 3535-3545. 
[33] D. W. Mackowski, A simplified model to predict the effects of aggregation on the absorption properties of soot particles, Journal of Quantitative Spectroscopy and Radiative Transfer 100 (2006) 237-249.

[34] F. Liu, G. J. Smallwood, Radiative properties of numerically generated fractal soot aggregates: the importance of configuration averaging, Journal of heat transfer 132 (2010) 023308.

[35] Ü. Ö. Köylü, G. Faeth, Spectral extinction coefficients of soot aggregates from turbulent diffusion flames, Journal of Heat Transfer 118 (1996) $415-421$.

[36] M. Schnaiter, H. Horvath, O. Möhler, K.-H. Naumann, H. Saathoff, O. Schöck, Uv-vis-nir spectral optical properties of soot and sootcontaining aerosols, Journal of Aerosol Science 34 (2003) 1421-1444.

[37] J. Yon, A. Bescond, F. Liu, On the radiative properties of soot aggregates part 1: Necking and overlapping, Journal of Quantitative Spectroscopy and Radiative Transfer 162 (2015) 197-206.

[38] H.-C. Chang, T. Charalampopoulos, Determination of the wavelength dependence of refractive indices of flame soot, Proc. R. Soc. Lond. A 430 (1990) 577-591.

[39] T. Nishizawa, N. Sugimoto, I. Matsui, A. Shimizu, B. Tatarov, H. Okamoto, Algorithm to retrieve aerosol optical properties from highspectral-resolution lidar and polarization mie-scattering lidar measurements, IEEE Transactions on Geoscience and Remote Sensing 46 (2008) 4094-4103.

[40] H. Li, C. Liu, L. Bi, P. Yang, G. W. Kattawar, Numerical accuracy of "equivalent" spherical approximations for computing ensemble-averaged scattering properties of fractal soot aggregates, Journal of Quantitative Spectroscopy and Radiative Transfer 111 (2010) 2127-2132.

[41] C. Sorensen, Light scattering by fractal aggregates: a review, Aerosol Science \& Technology 35 (2001) 648-687.

[42] Y. Zhao, L. Ma, Applicable range of the rayleigh-debye-gans theory for calculating the scattering matrix of soot aggregates, Applied optics 48 (2009) 591-597. 
[43] J. Dong, J. Zhao, L. Liu, Morphological effects on the radiative properties of soot aerosols in different internally mixing states with sulfate, Journal of Quantitative Spectroscopy and Radiative Transfer 165 (2015) $43-55$.

[44] F. Liu, J. Yon, A. Bescond, On the radiative properties of soot aggregates-part 2: Effects of coating, Journal of Quantitative Spectroscopy and Radiative Transfer 172 (2016) 134-145.

[45] K. Adachi, S. H. Chung, P. R. Buseck, Shapes of soot aerosol particles and implications for their effects on climate, Journal of Geophysical Research: Atmospheres 115 (2010).

[46] M. I. Mishchenko, L. Liu, D. W. Mackowski, T-matrix modeling of linear depolarization by morphologically complex soot and soot-containing aerosols, Journal of Quantitative Spectroscopy and Radiative Transfer 123 (2013) 135-144.

[47] Y. Wu, T. Cheng, L. Zheng, H. Chen, Effect of morphology on the optical properties of soot aggregated with spheroidal monomers, Journal of Quantitative Spectroscopy and Radiative Transfer 168 (2016) 158169.

[48] D. W. Mackowski, M. I. Mishchenko, Calculation of the t matrix and the scattering matrix for ensembles of spheres, JOSA A 13 (1996) 22662278 .

[49] N. Lu, C. Sorensen, Depolarized light scattering from fractal soot aggregates, Physical Review E 50 (1994) 3109.

[50] A. Bescond, J. Yon, F. Ouf, D. Ferry, D. Delhaye, D. Gaffié, A. Coppalle, C. Rozé, Automated determination of aggregate primary particle size distribution by tem image analysis: application to soot, Aerosol Science and Technology 48 (2014) 831-841.

[51] X. Ma, C. D. Zangmeister, J. Gigault, G. W. Mulholland, M. R. Zachariah, Soot aggregate restructuring during water processing, Journal of Aerosol Science 66 (2013) 209-219. 
[52] J. Luo, Y. Zhang, Q. Zhang, F. Wang, J. Liu, J. Wang, Sensitivity analysis of morphology on radiative properties of soot aerosols, Optics express 26 (2018) A420-A432.

[53] J. Yin, L. Liu, Influence of complex component and particle polydispersity on radiative properties of soot aggregate in atmosphere, Journal of Quantitative Spectroscopy and Radiative Transfer 111 (2010) 21152126 .

[54] N. Doner, F. Liu, Impact of morphology on the radiative properties of fractal soot aggregates, Journal of Quantitative Spectroscopy and Radiative Transfer 187 (2017) 10-19.

[55] A. J. Illingworth, H. Barker, A. Beljaars, M. Ceccaldi, H. Chepfer, N. Clerbaux, J. Cole, J. Delanoë, C. Domenech, D. P. Donovan, et al., The earthcare satellite: The next step forward in global measurements of clouds, aerosols, precipitation, and radiation, Bulletin of the American Meteorological Society 96 (2015) 1311-1332. 